\title{
BMJ Open Benefits of the addition of continuous or flash glucose monitoring versus standard practice using self-monitored blood glucose and haemoglobin A1c in the primary care of diabetes mellitus: a systematic review protocol
}

Alexander Kieu, ${ }^{1,2}$ Romona Devi Govender, ${ }^{1}$ Linda Östlundh, ${ }^{3}$ Jeffrey King ${ }^{1}{ }^{1}$

To cite: Kieu A, Govender RD, Östlundh L, et al. Benefits of the addition of continuous or flash glucose monitoring versus standard practice using self-monitored blood glucose and haemoglobin A1c in the primary care of diabetes mellitus: a systematic review protocol. BMJ Open 2021;11:e050027. doi:10.1136/ bmjopen-2021-050027

- Prepublication history and additional supplemental material for this paper are available online. To view these files, please visit the journal online (http://dx.doi.org/10.1136/ bmjopen-2021-050027).

Received 10 February 2021 Accepted 18 August 2021

D) Check for updates

(c) Author(s) (or their employer(s)) 2021. Re-use permitted under CC BY-NC. No commercial re-use. See rights and permissions. Published by BMJ.

${ }^{1}$ Department of Family Medicine, United Arab Emirates University, Al Ain, UAE

${ }^{2}$ Department of Family Medicine, Kanad Hospital, Al Ain, UAE

${ }^{3}$ National Medical Library, United Arab Emirates University, Al Ain, UAE

Correspondence to

Dr Jeffrey King;

jking@uaeu.ac.ae

\section{ABSTRACT}

Introduction Studies demonstrate that optimal glycaemic control reduces morbidity from diabetes mellitus but remains elusive in a significant portion of patients. Although research shows that continuous glucose monitoring (CGM) and flash glucose monitoring (FGM) improves glycaemic control in selected subsets of patients with diabetes in specialty practices, we found no systematic reviews evaluating the use of CGM/FGM in primary care, where the majority of patients with diabetes are cared for.

This systematic review aims to answer the questions: 'compared with usual care of self-monitoring blood glucose and haemoglobin $\mathrm{A} 1 \mathrm{c}(\mathrm{HbA1c})$, does the addition of CGM/FGM use in the primary care of patients with diabetes improve glycaemic control, decrease rates of hypoglycaemia, and improve patient and physician satisfaction?' and if so, 'what subgroups of primary care patients with diabetes are most likely to benefit?'. Methods and analysis Aligning with the Preferred Reporting Items for Systematic Review and Meta-Analysis Protocols guidelines, a search will be conducted in PubMed, EMBASE, Scopus, CINAHL, Cochrane Central Register of Controlled Trials and Web of Science. We will include studies investigating CGM/FGM use and reporting the primary outcome measure of $\mathrm{HbA} 1 \mathrm{c}$ and secondary outcome measures of hypoglycaemia, time in range, time below range, time above range and patient/staff satisfaction. We will examine which patient populations appear to benefit from CGM/FGM. Three independent researchers will use the Covidence systematic review software for blinded screening and study selection. The National Heart, Lung, and Blood Institute quality assessment tool and Grading of Recommendations Assessment, Development and Evaluation will be used to assess the risk of bias and quality of evidence.

Ethics and dissemination The systematic review methodology does not require ethics approval due to the nature of the study design. Study findings will be publicly available to a wide readership across disciplines and will be published in a peer-reviewed journal. PROSPERO registration number CRD42021229416.
Strengths and limitations of this study

- To our knowledge, this will be the first systematic review to analyse the clinical benefits of continuous glucose monitoring and flash glucose monitoring in the primary care setting.

- The evidence is located through a comprehensive and systematic search in seven biomedical and nursing databases.

- The Covidence systematic review software will be used for blinded screening, conflict resolving, extraction and quality assessment by three independent reviewers.

- National Heart, Lung, and Blood Institute and Grading of Recommendations Assessment, Development and Evaluation will be used to assess the risk of bias and quality of evidence.

- Limitations include an English language bias and exclusion of grey, non-peer-reviewed studies.

\section{INTRODUCTION}

Diabetes mellitus continues to be a major contributor to morbidity and mortality worldwide. Despite considerable expenditures of precious healthcare funds, outcomes continue to be quite poor, even in resourcerich countries with developed healthcare systems. Diabetes and impaired fasting glucose are strongly associated with a large increase in cardiovascular and all-cause mortality. ${ }^{12}$ Optimal glycaemic control has been shown in numerous studies to reduce the risk of morbidity, ${ }^{3}{ }^{4}$ but it has proved to be elusive in a significant portion of the patient population suffering from diabetes. ${ }^{5}$

Continuous glucose monitoring (CGM) and flash glucose monitoring (FGM) are technologies developed to add further precision to the monitoring and management of 
diabetes. Both CGM and FGM involve the use of subcutaneous sensors that sample interstitial glucose levels in the patient. In CGM, the sensor automatically sends glucose values to a receiver as often as every $5 \mathrm{~min},{ }^{6}$ and it can be used to alert the patient when the glucose trend is expected to exceed set limits, that is, result in hypoglycaemia or hyperglycaemia. Fingerstick calibration is required at least daily with $\mathrm{CGM}^{7}$ FGM, also called intermittently scanned CGM, uses similar approaches, but values are measured on demand, that is, the patient uses their smartphone or other devices to interrogate the sensor to measure the current interstitial glucose level, rather than having continuous measurements made. FGM does not require fingerstick calibration, as calibration is performed at the factory. ${ }^{8}$

Numerous studies and reviews have evaluated the use of CGM and FGM in caring for a selected subset of patients with diabetes, and they have shown some clinical benefit with regards to the secondary endpoint of goal-directed glycaemic control. ${ }^{9}$ This benefit has been demonstrated in both patients with either type $1^{10-13}$ or type 2 diabetes. ${ }^{1415}$ There is also evidence that the use of CGM in the management of pregnant patients with type 1 diabetes results in improved neonatal outcomes, ${ }^{16}$ and CGM may improve the glycaemic control of adolescents with type 1 diabetes. ${ }^{17}$ Although there is no recognised standard for what constitutes good glycaemic control, and most guidelines are now encouraging individualised haemoglobin A1c (HbA1c) goals based on patients' age, comorbidities and life expectancy, for the purposes of this review we will use the American Diabetes Association (ADA) guidelines 2019 for a goal A1c in most patients of less than $7 \%(53 \mathrm{mmol} / \mathrm{mol}, 154 \mathrm{mg} / \mathrm{dL}, 8.6 \mathrm{mmol} / \mathrm{L}){ }^{18}$

A major limiting factor in achieving glucose targets with current standard practice is hypoglycaemia with intensification of medical management. ${ }^{19}$ CGM/FGM have been shown to reduce episodes of both daytime and night-time hypoglycaemia. ${ }^{15} 2021$ Interestingly, a systematic review found that hypoglycaemia was very common in older adults treated with glucose-lowering agents, occurring in $28 \%-65 \%$ of participants in the included studies, most of whom $(80 \%-100 \%)$ were asymptomatic. ${ }^{22}$ Hypoglycaemia in this older patient population is associated with worsening cognitive impairment, dementia, ${ }^{23}$ falls, fractures, and cardiovascular complications, including death. ${ }^{24}$ Finally, recurrent hypoglycaemia can lead to impaired awareness of subsequent hypoglycaemic episodes. ${ }^{25}$ Although the use of CGM/FGM was not found in a systematic review to lead to restoration of awareness of hypoglycaemia, the review found that CGM can reduce rates of severe hypoglycaemia without worsening glycaemic control. ${ }^{26}$

Studies have highlighted that only consistent use of CGM yields improved clinical outcomes, ${ }^{27-29}$ which suggests patient satisfaction is clinically relevant. Although cutaneous complications with the use of CGM or FGM occur, less pain or discomfort was reported by most users of CGM/FGM than with the use of self-monitoring blood glucoses to test capillary blood glucose ${ }^{30}$ resulting in a high overall level of satisfaction with CGM. ${ }^{31}$

Surveys have shown that there is an insufficient number of endocrinologists to care for the large population of patients with diabetes, and in fact, the US primary care workforce cares for $85 \%$ of patients with diabetes according to data from a 2014 survey. ${ }^{32}$ However, although numerous studies have evaluated the use of CGM/FGM in specialty settings, our search showed there are no published systematic reviews for evaluating the use of CGM in the primary care setting. Thus, the purpose of this systematic review is to determine the benefits of CGM/FGM in the primary care of diabetes mellitus.

\section{Objectives}

This systematic review aims to answer the questions: 'compared with usual care of self-monitoring blood glucose and HbAlc, does the addition of CGM/FGM use in the primary care of patients with diabetes improve glycaemic control, decrease rates of hypoglycaemia, and improve patient and physician satisfaction?' and if so, 'what subgroups of primary care patients with diabetes are most likely to benefit?'.

\section{METHODS AND ANALYSIS}

This protocol follows the Preferred Reporting Items for Systematic Review and Meta-Analysis Protocols (PRISMA-P) guidelines ${ }^{33}$ and registered in the international prospective register of systematic reviews. Eventual amendments to this protocol will be recorded in the PROSPERO registration. It is anticipated that the study will commence in February 2021 and be completed by December 2021. A copy of the PRISMA-P checklist is available in online supplemental material 1 . The final review will be guided by the Cochrane Handbook for Systematic Reviews of Interventions ${ }^{34}$ and reported in accordance with the new Preferred Reporting Items for Systematic Reviews and Meta-Analyses (PRISMA) 2020 statement. $^{35}$

\section{Eligibility criteria}

The PICO (population, intervention, comparison, outcomes) required by the PRISMA format are as follows.

\section{Population}

Inclusion criteria are randomised and non-randomised controlled trials, non-controlled trials or observational studies with patients of any age with type 1 diabetes, type 2 diabetes or gestational diabetes under the care of a primary care provider, including those comanaged with an endocrinologist. Included studies will be grouped according to the type of diabetes assessed (type 1, type 2, both type 1 and 2 and gestational diabetes). We will exclude studies which were primarily focused on participants who were 
critically ill, hospitalised or comprised patients using closed loop systems.

\section{Intervention}

We will evaluate primary studies that report outcomes of the use of CGM or FGM in patients with diabetes in the primary care setting.

\section{Comparison}

The review seeks to compare the outcomes of the included studies on CGM/FGM with the practice of using HbA1c with or without self-monitoring of blood glucose levels in persons with diabetes in primary care practices.

\section{Outcomes}

Primary outcome

We have defined the primary outcome as the meeting of the ADA goals for glycaemic control in most patients of an HbA1c of $\leq 7 \%(\leq 53 \mathrm{mmol} / \mathrm{mol}, \leq 154 \mathrm{mg} / \mathrm{dL}$, $\leq 8.6 \mathrm{mmol} / \mathrm{L})$.

\section{Secondary outcomes}

We have included secondary outcomes of:

- Change from baseline HbA1c.

- Frequency of hypoglycaemia or $\leq 68 \mathrm{mg} / \mathrm{dL})$ and severe hypoglycaemia $(\leq 3.0 \mathrm{mmol} / \mathrm{L}$ or $\leq 54 \mathrm{mg} / \mathrm{dL})$.

- Hospitalisation.

- Time in target range (TIR).

- Time above target range (TAR).

- Time below target range (TBR). ${ }^{36}$

From our preliminary literature review, some studies used both self-monitoring of blood glucose (SMBG) and CGM/FGM in the control arm (but making clinical decisions based on SMBG) in order to compare change in TIR, TAR and TBR from baseline to study end to the intervention arm of CGM. However, for studies only evaluating change in TIR, TAR and TBR in the intervention arm without a control, we will assess the percentage change from baseline to study end in these outcomes.

For target ranges, we used a recent consensus report for patients with type 1 diabetes produced by the joint effort of multiple well-recognised organisations that defined the target range of $3.9-10.0 \mathrm{mmol} / \mathrm{L}(70.2-180 \mathrm{mg} / \mathrm{dL}){ }^{37}$ The goal of TIR $>70 \%$ for both patients with type 1 and type 2 diabetes $(>50 \%$ for 'older/high risk' individuals defined as cognitive deficits, renal disease, joint disease, osteoporosis, fracture, cardiovascular disease and/or those requiring assisted care) was defined by a different recent consensus report. ${ }^{38}$ For the subset of pregnant patients with diabetes, we will use the more stringent ADA guidelines. ${ }^{39}$ If data exist, we will report on glycaemic variability, patient-oriented clinically significant outcomes of patient satisfaction and diabetes-related complications such as major cardiovascular events, neuropathy, nephropathy and retinopathy.

\section{Type of studies}

We will include primary, peer-reviewed studies where the abstract or manuscript is available in the English language that evaluate the use of CGM or FGM in a primary care clinic regardless of the design of the study, with a minimum of 20 participants. We will exclude reviews, editorials, case reports, conference abstracts and comments.

\section{Information sources}

We will include the electronic databases PubMed, MEDLINE, EMBASE, Scopus, CINAHL, Cochrane Central Register of Controlled Trials and Web of Science in the search for literature. All databases will be searched from their inception to February 2021. A full search update will be performed ahead of manuscript submission.

\section{Search strategy}

A comprehensive, systematic search strategy was constructed by a medical librarian (LÖ) and reviewed by the subject specialists (AK, RDG and JK). Search terms were defined based on the PICO and the study inclusion and exclusion criteria as outlined above. PubMed and PubMed's MeSH was used in the pre-search phase in December-January 2021 to systematically identify and refine relevant search term variations and search techniques. The search strategy developed in PubMed will be adopted and applied in all selected databases without any geographical or publication year restrictions. We will use the 'Text Word' field (or similar) for the best possible information retrieval. Hand screening of the references lists of the included studies will also be conducted.

The search strategy developed through pre-searches in PubMed can be found in online supplemental material 2. A search $\log$ with notes, results and details for the searches in all included databases in addition to a PRISMA 2020 flow diagram outlining the details from the search and screening process will be appended to the final review. A search update in all databases will be conducted before finalising the manuscript to ensure the inclusion of relevant studies published after the search.

\section{Study records}

\section{Data management}

We will upload all records identified in the database search to Covidence systematic review software ${ }^{40}$ for automatic deduplication and blinded screening, conflict resolving, study selection and data extraction. Studies identified to be included in the review will be exported to EndNote, a citation management tool.

\section{Selection process}

The selection process in Covidence will follow the PRISMA workflow. Two independent reviewers (JK and RDG) will initially screen the title and abstracts of all identified papers based on the preset inclusion and exclusion criteria. A third reviewer (AK) will resolve eventual conflicts identified by the software. Full text of the papers identified as potentially eligible in the title and abstract screening will be reviewed independently by JK and AK. 
Reason for exclusion will be documented based on the preset study exclusion criteria imported to Covidence. RDG will resolve eventual conflicting exclusion reasons or paper inclusion/exclusion with the help of the software. Only the studies fulfilling the inclusion criteria will be fully screened for data extraction. The result from the screening and selection process will be documented in a PRISMA flow diagram.

\section{Data collection process}

Two independent reviewers (JK and AK) will extract data from the final studies identified eligible to be included in the review. The Covidence extraction module will be used to match the objectives of this study. Data to be extracted will include basic characteristics of the publication such as authors, year of publication, journal and country. Additionally, the study design, population, duration and CGM usage duration will be taken into consideration. The primary variables to be collected will be age; type of diabetes (type 1, type 2 or gestational); the use of CGM, FGM or both; episodes of hypoglycaemia as defined above in the Outcomes section of the PICO statement; the number of insulin injections given per day $(0,1$ or multiple); hospitalisation for complications of either diabetes or diabetes-related treatment; patient satisfaction scores and physician satisfaction scores. Quantitative data to be extracted include the weighted mean difference of change from baseline HbAlc, time in (TIR), above (TAR) and below (TBR) the goal range after CGM/FGM is used. Additionally, data on the relative risks of hospitalisations, hypoglycaemia and severe hypoglycaemia will be extracted.

Eventual discrepancies between the reviewers will be addressed with a third reviewer (RDG) and discussed until consensus is reached. Authors of the included papers will be contacted by email in case additional data are needed.

\section{Risk of bias in individual studies}

Two independent reviewers (JK and RDG) will evaluate the risk of bias and the quality of evidence of all included studies using the National Heart, Lung, and Blood Institute quality assessment tools. ${ }^{41}$ The reviewers will critically assess each study for its methodology to determine if any attrition, publication, outcome or conclusion biases are present. An appraisal of the quality of evidence for each of the outcomes will be given using the Grading of Recommendations Assessment, Development and Evaluation (GRADE) criteria. ${ }^{42}$ Eventual disagreements, unusual patterns or discrepancies in the assessment will be reviewed and discussed together with a third reviewer (AK) until consensus is reached. The existence of systematic differences in baseline characteristics between the groups compared in a study will be addressed in the discussion at the end of the study.

\section{Data synthesis}

In a comprehensive table, we will summarise the PICO characteristics of each study including all relevant interventions and outcomes. Thereafter we will determine which studies have similar characteristics and outcomes, which will allow us to group them and compare their relevant findings. We anticipate these subgroups to include type 1 diabetes, type 2 diabetes without insulin therapy, type 2 diabetes with only long-acting insulin therapy, type 2 diabetes with multiple daily insulin injections and gestational diabetes. Given our review will be inclusive of many study designs, we may group data according to their respective study design. Quantitative data to be reported for each study will include the mean HbAlc at study end of the intervention group (CGM/FGM) compared with control. We will then take the mean differences with the standard errors of the means, calculate the $95 \%$ CIs, and graphically represent our results. Weighted mean differences of change from baseline HbAlc, TIR, TAR and TBR after CGM/FGM intervention will similarly be analysed. Additionally, data on the relative risks of hospitalisations, hypoglycaemia and severe hypoglycaemia will be reported with their respective $95 \%$ CIs, along with qualitative data on patient and physician satisfaction. If any deviation from protocol plans is necessary, modifications to planned comparisons between studies will be made and noted. A narrative synthesis will be formulated for each subgroup of persons with diabetes, and we will give an appraisal of the utility of the addition of CGM or FGM, analysing which subgroups appear to benefit most from CGM/FGM.

If there are sufficient randomized controlled trials with low variability among them, a meta-analysis will be attempted comparing CGM/FGM to SMBG. A sensitivity analysis will be conducted by excluding studies with a high risk of bias, which will confirm the robustness of our results. We will assess heterogeneity using the $\mathrm{I}^{2}$ statistic, where $\geq 50 \%$ is considered heterogenous. ${ }^{42}{ }^{43}$ If heterogeneity is determined, a meta-regression analysis will be performed to evaluate whether baseline HbAlc, duration of CGM/FGM use, and study design influence CGM/ FGM's effects on HbAlc. Egger's regression test will be used to detect publication bias. ${ }^{44}$

\section{Strength of evidence}

We will use the GRADE ${ }^{45}$ tool to determine the strength of evidence, including publication bias, indirectness, inconsistencies, imprecision and risk of bias. Two independent reviewers (JK and $\mathrm{AK}$ ) will evaluate the quality of each study, and a third independent reviewer (RDG) will resolve any potential conflict.

\section{Patient and public involvement}

No patient or members of the public will be included in the performance of this study.

\section{DISCUSSION}

This systematic review will be conducted to compare the benefits of adding CGM/FGM to usual care in the monitoring and management of diabetes mellitus in primary 
care. This technology has shown promise among persons with type 1 diabetes, and a systematic review and metaanalysis by Park and Le in 2018 demonstrated that CGM can have a modest improvement in HbAlc in type 2 diabetes. ${ }^{6}$ However, it is unclear whether these benefits would be realised in the primary care setting where many persons with either type 1 or type 2 diabetes seek medical care. This review will allow us to determine whether the addition of CGM/FGM will lead to better outcomes in achieving glycaemic control while avoiding frequent hypoglycaemia, both of which have been strongly associated with reduced morbidity and mortality.

\section{Ethics and dissemination}

Considering the study design of systematic reviews, ethics approval is not needed. We intend to publish the review in a peer-reviewed journal where the results will be available in an open access format.

Contributors AK is the primary author for the study. The study design and draft of the protocol was conducted by AK, JK, RDG and LÖ. JK is the corresponding author. LÖ developed the search strategy and provided feedback on the study protocol design. The final manuscript was approved by all authors.

Funding The authors have not declared a specific grant for this research from any funding agency in the public, commercial or not-for-profit sectors.

Competing interests None declared.

Patient consent for publication Not required.

Provenance and peer review Not commissioned; externally peer reviewed.

Supplemental material This content has been supplied by the author(s). It has not been vetted by BMJ Publishing Group Limited (BMJ) and may not have been peer-reviewed. Any opinions or recommendations discussed are solely those of the author(s) and are not endorsed by BMJ. BMJ disclaims all liability and responsibility arising from any reliance placed on the content. Where the content includes any translated material, BMJ does not warrant the accuracy and reliability of the translations (including but not limited to local regulations, clinical guidelines, terminology, drug names and drug dosages), and is not responsible for any error and/or omissions arising from translation and adaptation or otherwise.

Open access This is an open access article distributed in accordance with the Creative Commons Attribution Non Commercial (CC BY-NC 4.0) license, which permits others to distribute, remix, adapt, build upon this work non-commercially, and license their derivative works on different terms, provided the original work is properly cited, appropriate credit is given, any changes made indicated, and the use is non-commercial. See: http://creativecommons.org/licenses/by-nc/4.0/.

\section{ORCID iD}

Jeffrey King http://orcid.org/0000-0003-4974-9904

\section{REFERENCES}

1 Barr ELM, Zimmet PZ, Welborn TA, et al. Risk of cardiovascular and all-cause mortality in individuals with diabetes mellitus, impaired fasting glucose, and impaired glucose tolerance: the Australian diabetes, obesity, and lifestyle study (Ausdiab). Circulation 2007;116:151-7.

2 Cavalot F, Pagliarino A, Valle M, et al. Postprandial blood glucose predicts cardiovascular events and all-cause mortality in type 2 diabetes in a 14-year follow-up: lessons from the san luigi gonzaga diabetes study. Diabetes Care 2011;34:2237-43.

3 Diabetes Control and Complications Trial Research Group, Nathan DM, Genuth S, et al. The effect of intensive treatment of diabetes on the development and progression of long-term complications in insulin-dependent diabetes mellitus. N Engl J Med 1993;329:977-86.

4 UK Prospective Diabetes Study (UKPDS) Group. Intensive bloodglucose control with sulphonylureas or insulin compared with conventional treatment and risk of complications in patients with type 2 diabetes (UKPDS 33). UK prospective diabetes study (UKPDS) group. Lancet 1998;352:837-53.

5 Edelman SV, Polonsky WH. Type 2 diabetes in the real world: the elusive nature of glycemic control. Diabetes Care 2017;40:1425-32.

6 Park C, Le QA. The effectiveness of continuous glucose monitoring in patients with type 2 diabetes: a systematic review of literature and meta-analysis. Diabetes Technol Ther 2018;20:613-21.

7 Klonoff DC, Ahn D, Drincic A. Continuous glucose monitoring: a review of the technology and clinical use. Diabetes Res Clin Pract 2017;133:178-92.

8 Heinemann L, Freckmann G, versus FGM CGM. CGM versus FGM; or, continuous glucose monitoring is not flash glucose monitoring. $J$ Diabetes Sci Technol 2015;9:947-50.

9 Evans M, Welsh Z, Ells S, et al. The impact of flash glucose monitoring on glycaemic control as measured by HbA1c: a metaanalysis of clinical trials and real-world observational studies. Diabetes Ther 2020;11:83-95.

10 Beck RW, Riddlesworth T, Ruedy K, et al. Effect of continuous glucose monitoring on glycemic control in adults with type 1 diabetes using insulin injections: the diamond randomized clinical trial. JAMA 2017;317:371-8.

11 Lind M, Polonsky W, Hirsch IB, et al. Continuous glucose monitoring vs conventional therapy for glycemic control in adults with type 1 diabetes treated with multiple daily insulin injections: the gold randomized clinical trial. JAMA 2017;317:379-87.

12 Campbell FM, Murphy NP, Stewart C, et al. Outcomes of using flash glucose monitoring technology by children and young people with type 1 diabetes in a single arm study. Pediatr Diabetes 2018;19:1294-301.

13 Deiss D, Bolinder J, Riveline J-P, et al. Improved glycemic control in poorly controlled patients with type 1 diabetes using real-time continuous glucose monitoring. Diabetes Care 2006;29:2730-2.

14 Beck RW, Riddlesworth TD, Ruedy K, et al. Continuous glucose monitoring versus usual care in patients with type 2 diabetes receiving multiple daily insulin injections: a randomized trial. Ann Intern Med 2017;167:365-74.

15 Ida S, Kaneko R, Murata K. Utility of real-time and retrospective continuous glucose monitoring in patients with type 2 diabetes mellitus: a meta-analysis of randomized controlled trials. $J$ Diabetes Res 2019;2019:1-10.

16 Feig DS, Donovan LE, Corcoy R, et al. Continuous glucose monitoring in pregnant women with type 1 diabetes (CONCEPTT): a multicentre international randomised controlled trial. Lancet 2017;390:2347-59.

17 Dorando E, Haak T, Pieper D. Continuous glucose monitoring for glycaemic control in children and adolescents diagnosed with diabetes type 1: a systematic review and meta-analysis. Exp Clin Endocrinol Diabetes 2021.

18 American Diabetes Association. Standards of medical care in diabetes-2019. Diabetes Care 2019;42:s1-2 https://care. diabetesjournals.org/content/42/Supplement_1

19 Cryer PE. Hypoglycaemia: the limiting factor in the glycaemic management of type I and type II diabetes. Diabetologia 2002;45:937-48.

20 Bolinder J, Antuna R, Geelhoed-Duijvestijn P, et al. Novel glucosesensing technology and hypoglycaemia in type 1 diabetes: a multicentre, non-masked, randomised controlled trial. Lancet 2016;388:2254-63.

21 Haak T, Hanaire H, Ajjan R, et al. Flash glucose-sensing technology as a replacement for blood glucose monitoring for the management of insulin-treated type 2 diabetes: a multicenter, open-label randomized controlled trial. Diabetes Ther 2017;8:55-73.

22 Mattishent K, Loke YK. Detection of asymptomatic drug-induced hypoglycemia using continuous glucose monitoring in older peoplesystematic review. J Diabetes Complications 2018;32:805-12.

23 Mattishent K, Loke YK. Bi-Directional interaction between hypoglycaemia and cognitive impairment in elderly patients treated with glucose-lowering agents: a systematic review and metaanalysis. Diabetes Obes Metab 2016;18:135-41.

24 Mattishent K, Loke YK. Meta-analysis: association between hypoglycaemia and serious adverse events in older patients. $J$ Diabetes Complications 2016;30:811-8.

25 Lingenfelser T, Renn W, Sommerwerck U, et al. Compromised hormonal counterregulation, symptom awareness, and neurophysiological function after recurrent short-term episodes of insulin-induced hypoglycemia in IDDM patients. Diabetes 1993;42:610-8.

26 Yeoh E, Choudhary P, Nwokolo M, et al. Interventions that restore awareness of hypoglycemia in adults with type 1 diabetes: a systematic review and meta-analysis. Diabetes Care 2015;38:1592-609. 
27 Hirsch IB, Abelseth J, Bode BW, et al. Sensor-augmented insulin pump therapy: results of the first randomized treat-to-target study. Diabetes Technol Ther 2008;10:377-83.

28 Garg S, Zisser H, Schwartz S, et al. Improvement in glycemic excursions with a transcutaneous, real-time continuous glucose sensor: a randomized controlled trial. Diabetes Care 2006;29:44-50.

29 , Tamborlane WV, Beck RW, et al, Juvenile Diabetes Research Foundation Continuous Glucose Monitoring Study Group. Continuous glucose monitoring and intensive treatment of type 1 diabetes. N Engl J Med 2008;359:1464-76.

30 Asarani NAM, Reynolds AN, Boucher SE, et al. Cutaneous complications with continuous or flash glucose monitoring use: systematic review of trials and observational studies. J Diabetes Sci Technol 2020;14:328-37.

31 Bruttomesso D, Laviola L, Avogaro A, et al. The use of real time continuous glucose monitoring or flash glucose monitoring in the management of diabetes: a consensus view of Italian diabetes experts using the delphi method. Nutrition, Metabolism and Cardiovascular Diseases 2019;29:421-31.

32 Vigersky RA, Fish L, Hogan P, et al. The clinical endocrinology workforce: current status and future projections of supply and demand. J Clin Endocrinol Metab 2014;99:3112-21.

33 Moher D, Shamseer L, Clarke M, et al. Preferred reporting items for systematic review and meta-analysis protocols (PRISMA-P) 2015 statement. Syst Rev 2015;4:1.

34 Higgins JPT, Thomas J, Chandler J. Cochrane Handbook for systematic reviews of interventions version 6.2, 2021. www.training. cochrane.org/handbook

35 Page MJ, Moher D, Bossuyt PM, et al. PRISMA 2020 explanation and elaboration: updated guidance and exemplars for reporting systematic reviews. BMJ 2021:372:160.
36 Advani A. Positioning time in range in diabetes management. Diabetologia 2020;63:242-52.

37 Agiostratidou G, Anhalt H, Ball D, et al. Standardizing clinically meaningful outcome measures beyond $\mathrm{HbA}$ for type 1 diabetes: a consensus report of the American association of clinical endocrinologists, the American association of diabetes educators, the American diabetes association, the endocrine society, JDRF International, The Leona M. and Harry B. helmsley charitable trust, the pediatric endocrine society, and the T1D exchange. Diabetes Care 2017; 40:1622-30.

38 Battelino T, Danne T, Bergenstal RM, et al. Clinical targets for continuous glucose monitoring data interpretation: recommendations from the International consensus on time in range. Diabetes Care 2019;42:1593-603.

39 American Diabetes Association. 14. Management of diabetes in pregnancy: standards of medical care in diabetes-2019. Diabetes Care 2019;42:S165-72.

40 Veritas Health Innovation. Covidence systematic review software, 2020. Available: https://www.covidence.org

41 National Heart, Lung and Blood Institute. Study quality assessment tools. Available: https://www.nhlbi.nih.gov/health-topics/studyquality-assessment-tools [Accessed 24 Jun 2021].

42 Higgins JPT, Thompson SG, Deeks JJ, et al. Measuring inconsistency in meta-analyses. BMJ 2003;327:557-60.

43 Higgins JPT, Thompson SG. Quantifying heterogeneity in a metaanalysis. Stat Med 2002;21:1539-58.

44 Egger M, Davey Smith G, Schneider M, et al. Bias in meta-analysis detected by a simple, graphical test. BMJ 1997;315:629-34.

45 Siemieniuk R, Guyatt G. Evidence based medicine (EBM) toolkit: what is GRADE? [Internet]. Available: https://bestpractice.bmj.com/ info/toolkit/learn-ebm/what-is-grade/ [Accessed 02 Feb 2021]. 